\title{
Micro-Vortex Generators on Transonic Convex-Corner Flow
}

\author{
Kung-Ming Chung ${ }^{1, *(\mathbb{D},}$ Kao-Chun $\mathrm{Su}^{2}$ and Keh-Chin Chang ${ }^{2} \mathbb{(}$ \\ 1 Aerospace Science and Technology Research Center, National Cheng Kung University, Tainan 711, Taiwan \\ 2 Department of Aeronautics and Astronautics, National Cheng Kung University, Tainan 710, Taiwan; \\ P48061463@ncku.edu.tw (K.-C.S.); kcchang@mail.ncku.edu.tw (K.-C.C.) \\ * Correspondence: kmchung@mail.ncku.edu.tw; Tel.: +886-6-239281
}

Citation: Chung, K.-M.; Su, K.-C.;

Chang, K.-C. Micro-Vortex

Generators on Transonic

Convex-Corner Flow. Aerospace 2021,

8, 268. https://doi.org/10.3390/

aerospace 8090268

Academic Editor: Konstantinos

Kontis

Received: 16 August 2021

Accepted: 16 September 2021

Published: 17 September 2021

Publisher's Note: MDPI stays neutral with regard to jurisdictional claims in published maps and institutional affiliations.

\begin{abstract}
A convex corner models the upper surface of a deflected flap and shock-induced boundary layer separation occurs at transonic speeds. This study uses micro-vortex generators (MVGs) for flow control. An array of MVGs (counter-rotating vane type, ramp type and co-rotating vane type) with a height of $20 \%$ of the thickness of the incoming boundary layer is installed upstream of a convex corner. The surface pressure distributions are similar regardless of the presence of MVGs. They show mild upstream expansion, a strong favorable pressure gradient near the corner's apex and downstream compression. A corrugated surface oil flow pattern is observed in the presence of MVGs and there is an onset of compression moving downstream. The counter-rotating vane type MVGs produce a greater reduction in peak pressure fluctuations and the ramp type decreases the separation length. The presence of MVGs stabilizes the shock and shock oscillation is damped.
\end{abstract}

Keywords: transonic flow; convex corner; micro-vortex generator; shock-induced boundary layer separation

\section{Introduction}

A deflected trailing-edge flap increases camber and lift at a constant angle of attack (or lift-to-drag ratio) for transport aircraft. There is also a higher buffet boundary (structural response due to pressure fluctuations) and a greater pitching moment [1]. In a strong adverse pressure gradient (or shock region) at transonic speeds, flap deflection leads to boundary layer separation when the critical Mach number $(\approx 1.3)$ is achieved [2]. A convex corner can be used to model the upper surface of a deflected flap. The typical mean surface pressure pattern for a turbulent boundary layer over a convex corner was determined by Chung [3]. There is initial mild upstream expansion, a strong favorable pressure gradient near the corner's apex and downstream compression $(\lambda$ shock structure at transonic speeds, as shown in Figure 1). The flow characteristics are dominated by the freestream Mach number, $M$, and angle of the convex corner, $\eta$. A similarity parameter $\beta$ $\left(=M^{2} \eta / \sqrt{1-M^{2}}\right)$ was proposed for compressible flow around a convex corner, which involves transition from subsonic to transonic flow $(\beta \approx 8)$, shock-induced boundarylayer separation (SIBLS) $(\beta>13)$ and peak pressure fluctuations [4]. These peak pressure fluctuations are associated with low-frequency, large-scale shock oscillation in the range of several hundred hertz to several kilohertz [5].

SIBLS significantly reduces aerodynamic efficiency and increases energy loss [6-8]. Passive vortex generators (VGs) with a height, $h$, that is of the order of the thickness of the incoming boundary layer, $\delta$, are used to control boundary layer separation [9-12]. The geometric factors for VGs also include length, spacing and positions, but the effect of $h$ is the most significant [13]. The device is typically normal to the surface and is positioned at an angle of incidence, $\alpha$, to the local flow, to produce streamwise vortices in the flow close to the surface [14]. Therefore, VGs that are located upstream of the flow separation energize the boundary layer or increase near-wall momentum, which is transferred from the outer flow (or wall-ward momentum transfer). 


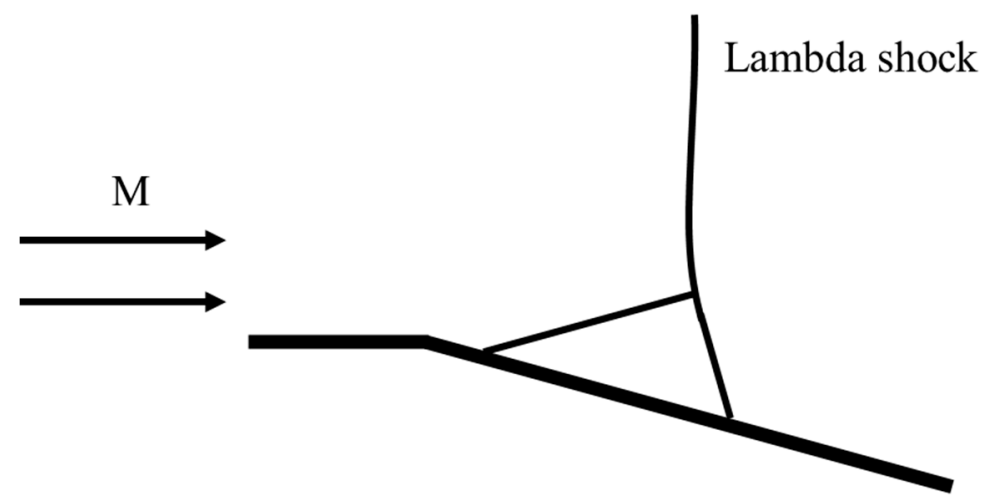

Figure 1. A schematically drawing of $\lambda$-shock structure over the region of convex corner.

Sub- $\delta$-scale or micro VGs $\left(h / \delta=h^{*}<1\right.$, MVGs) that have a rectangular, delta or trapezoidal shape ensure effective mixing over a region that is several times their own height [15-18] and device-induced streamwise vortices may last up to $h^{*}$ of 100 [13]. MVGs also induce less device (or parasitic) drag than larger scale VGs.

MVGs with counter-rotating vanes (CRV), co-rotating vanes (Vane) or ramps (Ramp) are most commonly used to control boundary-layer separation [14]. For CRV MVGs, vanes are grouped in pairs and produce vortices of alternating sign, as shown in Figure 2. There is a significant reduction in separation-induced fluctuations in shear stress on the upper surface for a high-lift airfoil [15]. Vane MVGs are the most common type of VG which energizes the low-momentum boundary layer flow near the surface. Large vortical structures and up-sweep flows are attenuated [19]. The amplitude of the forced shock wave oscillation is also reduced by delaying the upstream displacement of the leading shock [20]. In the presence of Ramp MVGs, a horseshoe vortex system and a vortex pair form and the velocity is greater close to the surface (unsteady wake trailing downstream), so energetic air is transferred from the primary vortex pair [21,22].

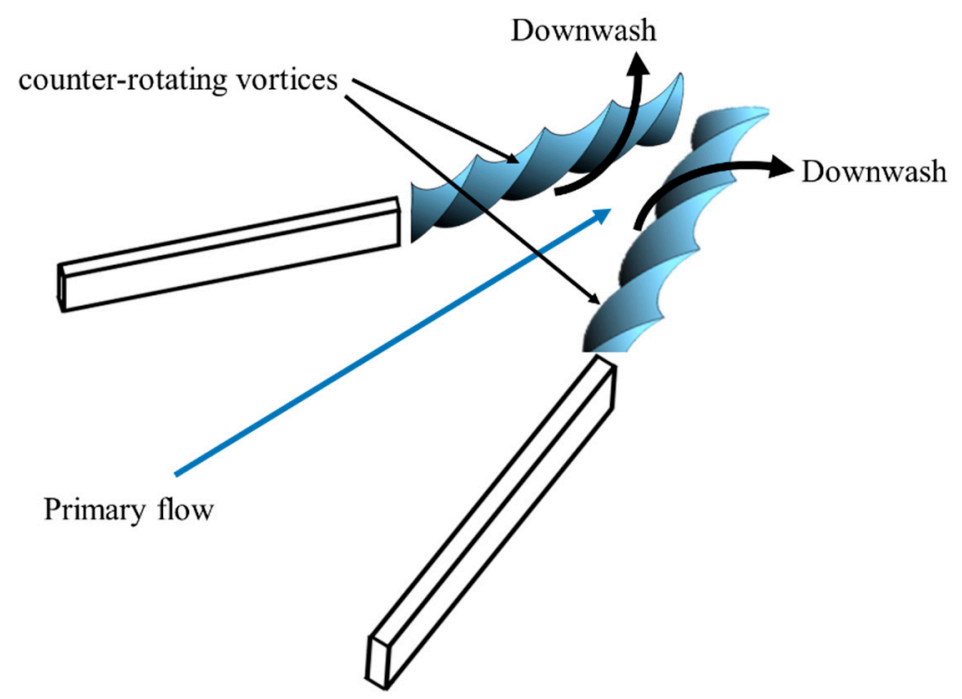

Figure 2. 3D flow structure of a CRV type MVG.

A convex corner is used to model the upper surface of a deflected flap for an increase in lift. In the transonic flow regime, SIBLS occurs for $\beta>13$, which results in peak pressure fluctuations due to shock oscillation [23]. This affects aerodynamic characteristics for flight vehicles, such as wave drag and buffet boundary. Shock wave control technologies (MVGs, bleeding or suction) can be used to minimize the adversarial effects of SIBLS. This study uses MVGs (CRV, Ramp and Vane types) with a value of $h^{*}=0.2$ to alleviate flow separation. The effectiveness of MVGs is determined using surface mean/fluctuating 
pressure measurements and surface oil flow visualization. Before discussing the results, details of the experiment setup are outlined next.

\section{Experimental Setup}

\subsection{Transonic Wind Tunnel}

This experiment was conducted in the blowdown transonic wind tunnel, as shown in Figure 3, at the Aerospace Science and Technology Research Center in National Cheng Kung University (ASTRC/NCKU). The facility consists of compressors, air dryers (dew point $\left.\approx-40{ }^{\circ} \mathrm{C}\right)$, a cooling water system, three air storage tanks $\left(180 \mathrm{~m}^{3}\right)$ and a tunnel. The constant-area test section has a cross-sectional area of $600 \mathrm{~mm} \times 600 \mathrm{~mm}$ and a length of $1500 \mathrm{~mm}$. The test section was assembled with solid sidewalls for a reduction in background noise and perforated top/bottom walls with $6 \%$ porosity. Holes were inclined at $60^{\circ}$ to minimize the wall interference. The operating Mach number ranges from 0.2 to 1.4. The stagnation pressure, $P_{0}$, is $172 \pm 0.5 \mathrm{kPa}$ and the stagnation temperature, $T_{0}$, is $28-32{ }^{\circ} \mathrm{C}$. The unit Reynolds number has a maximum value of $2 \times 10^{7} / \mathrm{m}$. The respective flow angularity and the centerline Mach number uniformity in the test section are $0.2^{\circ}$ and 0.005 .

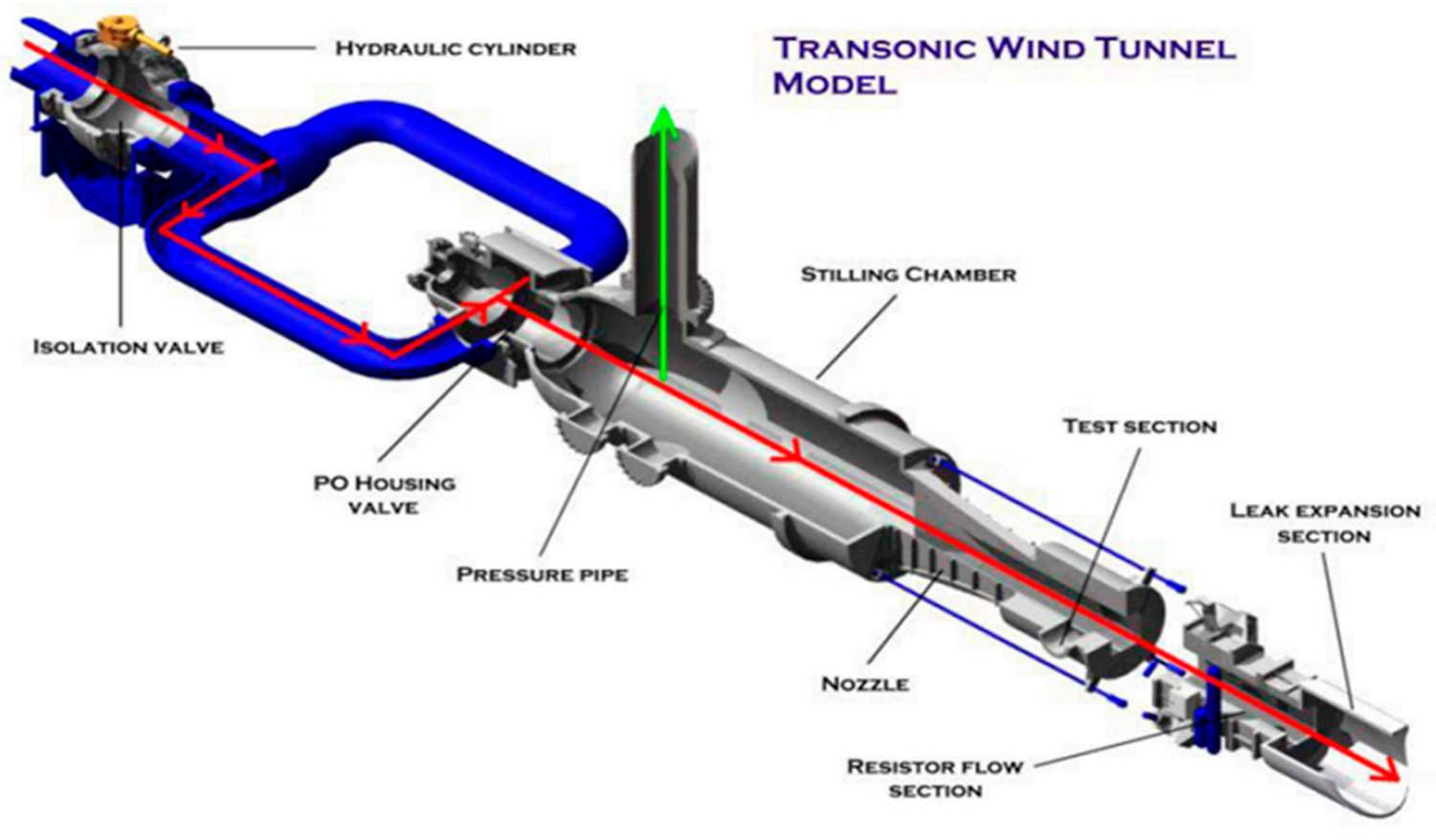

Figure 3. Transonic wind tunnel at ASTRC/NCKU.

\subsection{Test Model}

The test model consists of a flat plate and an interchangeable instrumentation plate with/without an array of MVGs, as shown in Figure 4. The flat plate with a leading edge of $3^{\circ}$ is $450 \mathrm{~mm}$ long and $150 \mathrm{~mm}$ wide for a naturally developed, turbulent boundary layer [24]. The interchangeable instrumentation plate is $170 \mathrm{~mm}$ long and $150 \mathrm{~mm}$ wide. A sharp convex corner with an angle, $\eta$, of $13^{\circ}$ or $15^{\circ}$ is located $500 \mathrm{~mm}$ from the leading edge of the flat plate. Two side fences of $(13.5 \times 4.5 \times 0.5 \mathrm{~cm})$ were also installed at both sides of the instrumentation plate to prevent crossflow. The freestream Mach number, $M$, is 0.83 and $0.89 \pm 0.01$. The value of $\delta$ at $25 \mathrm{~mm}$ upstream of the convex corner is approximately $7 \mathrm{~mm}[23,25]$. The Reynolds number based on the boundary layer thickness, $R e_{\delta}$, is 1.63 and $1.69 \times 10^{5}$. The baseline experiment, denoted as the baseline case, does not use MVGs. 


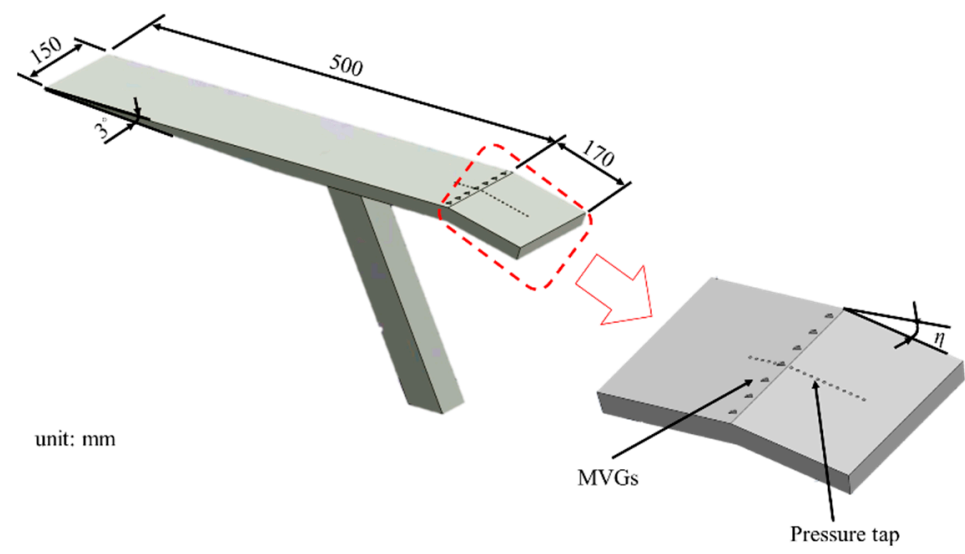

Figure 4. Test configuration.

The rear face of an array of MVGs is located $3 \mathrm{~mm}$ upstream of the convex corner. Seventeen pressure taps were machined perpendicular to the surface of the test model along the centerline: 5 pressure taps ahead of the MVGs and 12 pressure taps downstream of the corner. The spacing between the pressure taps was $6 \mathrm{~mm}$. Three types of MVGs were used: CRV, Vane and Ramp. Sketches of the MVGs are shown in Figure 5 and the parameters for the MVGs are shown in Table 1. For all MVGs, the length, $l$, and height, $h$, are $1 \delta$ and $0.2 \delta$ (lower device drag), respectively. The width for the CRV and Vane types, $w_{v}$, is $0.2 \delta$ and for the Ramp type, $w_{r}$, is $0.5 \delta$. The spacing, $D$, between MVGs is $3 \delta$, which corresponds to an array with seven MVGs. For the CRV type, the front, $d$, and rear, $s$, spacing are $1 \delta$ and $0.5 \delta$, respectively. The value of $\alpha$ is $15^{\circ}$.

(a)

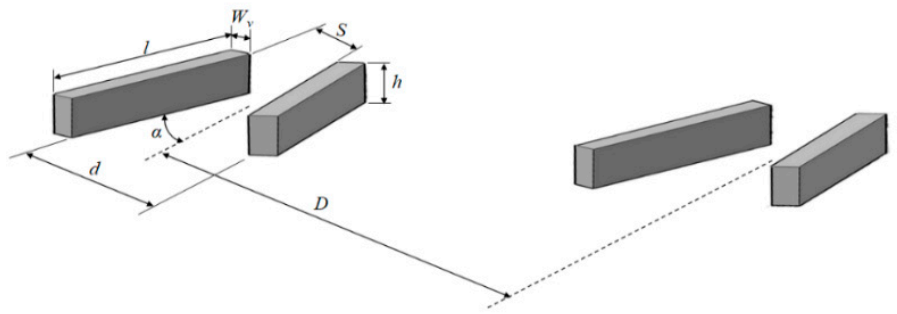

(b)

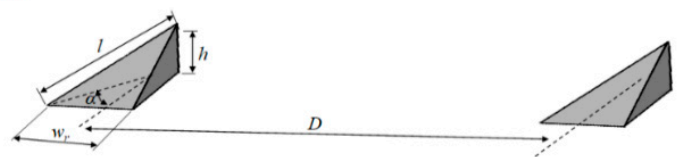

(c)

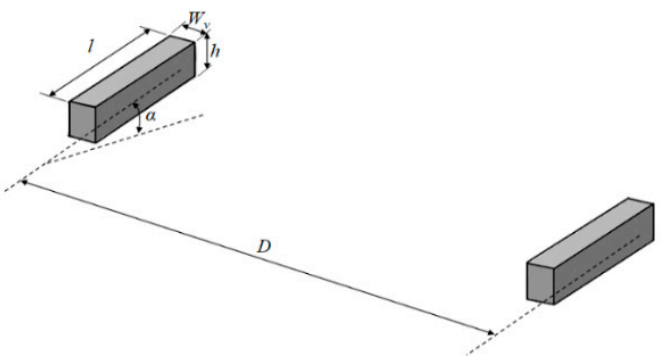

Figure 5. Configuration of MVGs: (a) counter-rotating vane type, (b) ramp type and (c) co-rotating vane type. 
Table 1. Geometry of the MVGs.

\begin{tabular}{cc}
\hline Parameters & Value \\
\hline$h / \delta$ & 0.2 \\
$l / \delta$ & 1.0 \\
$D / \delta$ & 3.0 \\
$w_{v} / \delta$ & 0.2 \\
$w_{r} / \delta$ & 0.5 \\
$s / \delta$ & 0.5 \\
$d / \delta$ & 1.0 \\
$\alpha$ & $15^{\circ}$ \\
\hline
\end{tabular}

\subsection{Instrumentation and Data Acquisition System}

Flush-mounted Kulite pressure transducers (Model XCS-093-25A, B screen) with a natural frequency of $200 \mathrm{kHz}$ were used to measure the mean, $P_{w}$, and fluctuating, $\sigma_{p}$, surface pressures. The nominal outer diameter of the Kulite sensor is $2.36 \mathrm{~mm}$ and the diameter of its pressure-sensitive element is $0.97 \mathrm{~mm}$. Figure 6 shows that external amplifiers (Ectron Model 753A) with a roll-off frequency of approximately $140 \mathrm{kHz}$ were used to improve the signal-to-noise ratio. A National Instruments (CI SCXI) was used to trigger all input channels and to record data. An experiment for a flat plat using $10 \mathrm{Kulite}$ sensors was conducted to determine the experimental uncertainty. The uncertainty for the mean surface pressure coefficient, $P_{w} / P_{o}$, and the surface fluctuating pressure coefficient, $\sigma_{p} / P_{w}$, is respectively determined using the standard deviation of surface pressure and the mean value of surface pressure fluctuations for 10 Kulite sensors. The respective value for $P_{w} / P_{o}$ and $\sigma_{p} / P_{w}$ is $1.24 \%$ and $0.97 \%$.

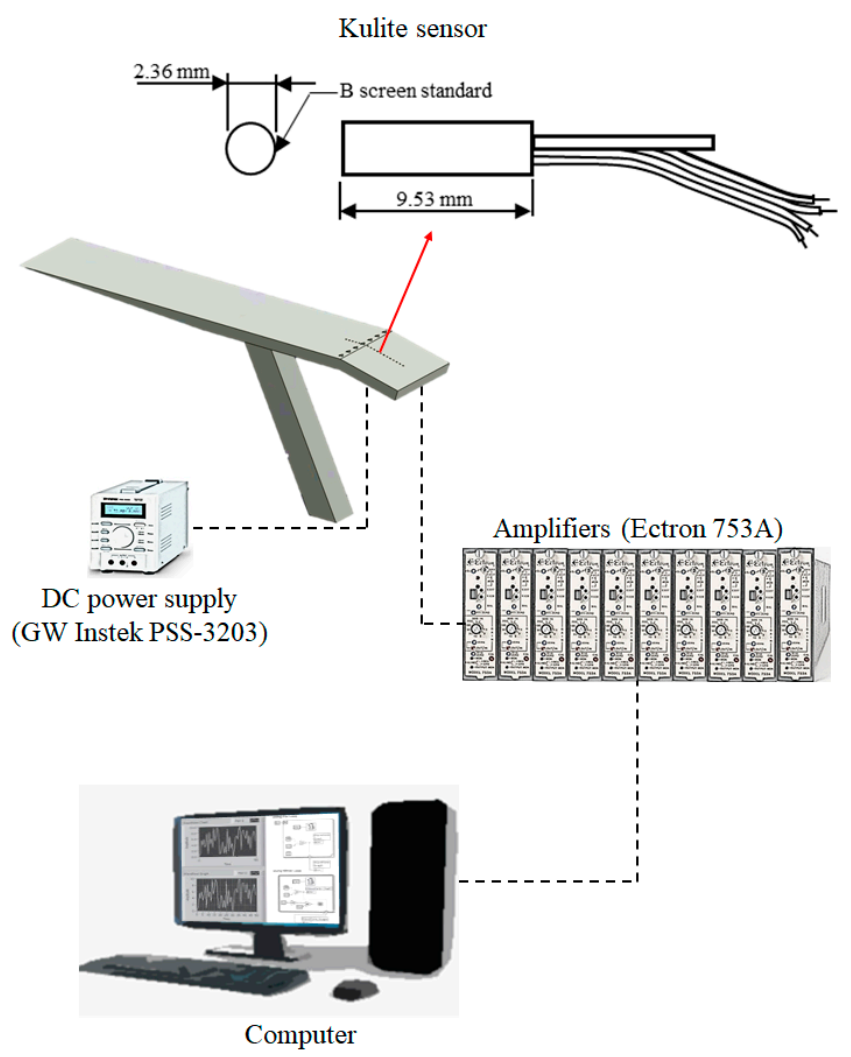

Figure 6. Experimental setup.

Shock oscillation occurs in shock wave/boundary layer interactions. An unsteady shock moving forward and backward across a pressure sensor results in intermittent pressure signals, as shown in Figure 7a. A two-threshold method (THM, a conditional 
analysis technique) [26] was used to determine shock zero-crossing frequency, $f_{s}$, where $T_{1}$ (the lower threshold) $=P_{w}+3 \sigma_{p}$ and $T_{2}$ (the upper threshold) $=P_{w}+6 \sigma_{p}$, as shown in Figure 7a. $P_{w}>T_{2}$ represents the flow downstream of the shock. The counter records the time between successive shock waves until $P_{w}<T_{1}$. The time between consecutive passages of the shock over the pressure transducer (shock motion) is used to calculate $f_{s}$ which determines the unsteady characteristics of the separation shock wave motion. Figure $7 \mathrm{~b}$ shows the conversion of the pressure signal into a boxcar of amplitude unity and varying frequency.

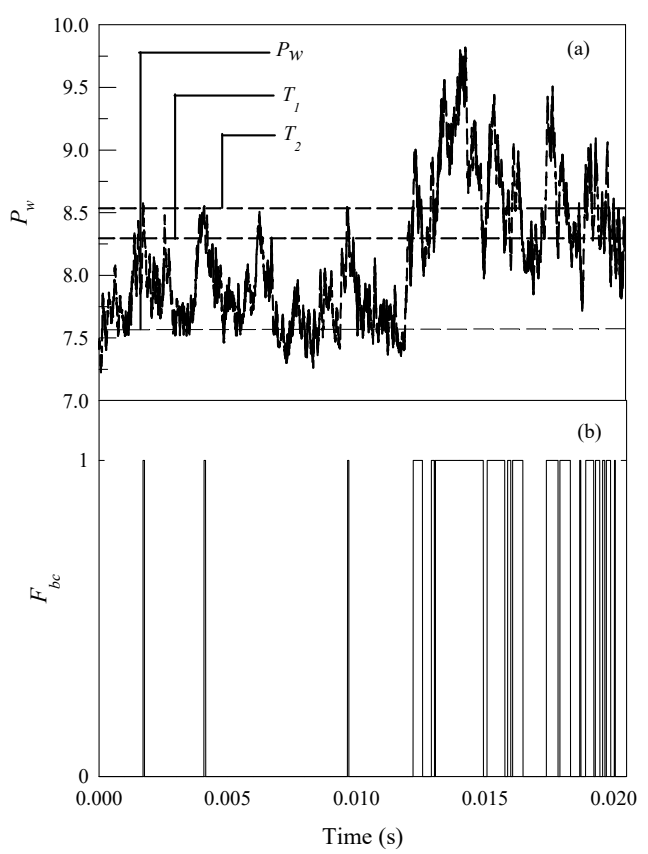

Figure 7. (a) Pressure signal; (b) boxcar using the THM.

Oil-flow visualization technique was used to determine the surface flow pattern. A mixture of titanium dioxide, oil, oleic acid and kerosene was applied to the surface of instrumentation plate. If there is shock-induced boundary layer separation, titanium dioxide accumulates at the separation position. The end of the deflected streamlines is denoted as the reattachment position.

\section{Results and Discussion}

\subsection{Mean Surface Pressure Distributions}

At $M=0.83$, the mean surface pressure distributions along the centerline of the instrumentation plate for $\eta=13^{\circ}$ and $15^{\circ}$ are shown in Figure 8. For the baseline case, they respectively correspond to near incipient separation and intense shock oscillation [3]. The vertical axis represents $P_{w} / P_{o}$ and the horizontal axis corresponds to the normalized streamwise location, $X^{*}(=X / \delta)$. The origin of the horizontal axis is at the corner's apex and there is no data for $X^{*}=-2$ and 0 because of the presence of MVGs. The sonic condition $\left(P_{w} / P_{o}=0.5283\right)$ is also shown for reference. The $P_{w} / P_{o}$ distributions with/without MVGs are similar in that they show upstream mild expansion, strong expansion near the corner's apex and downstream compression. The presence of MVGs has a minor effect on the amplitude of $P_{w} / P_{o}$ upstream of the corner. 


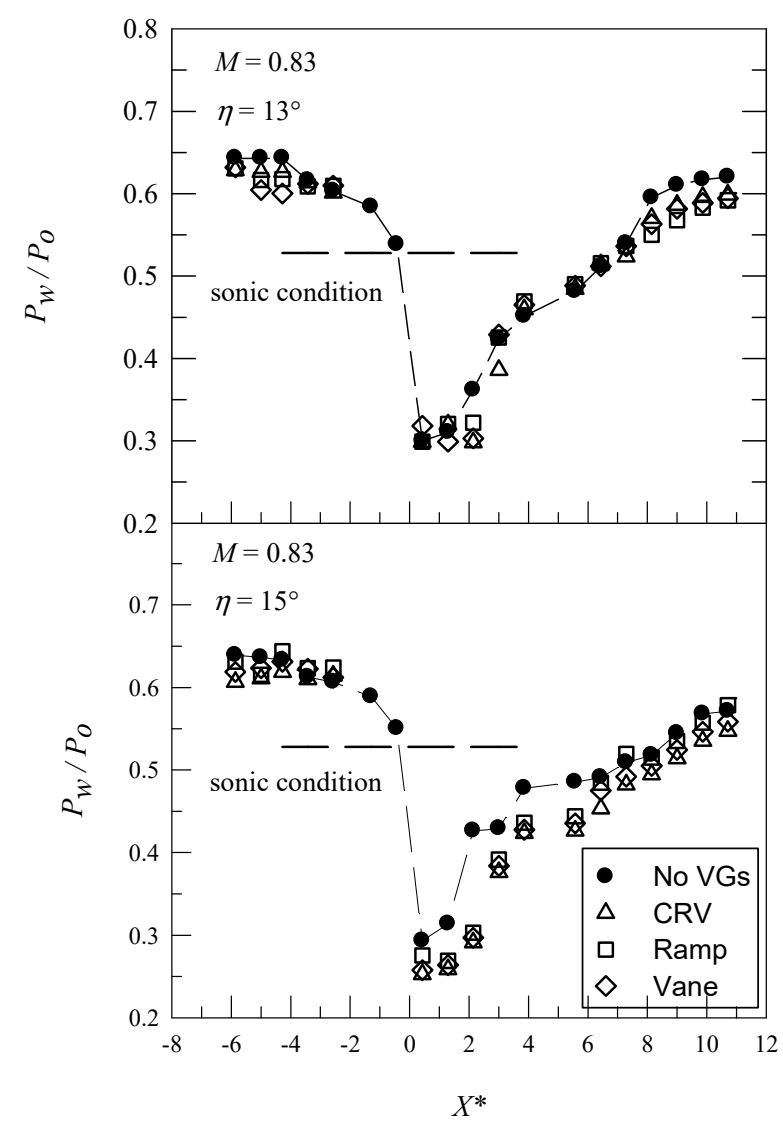

Figure 8. Mean surface pressure distributions for $M=0.83$.

For $\eta=13^{\circ}$, the minimum value for $P_{w} / P_{o},\left(P_{w} / P_{o}\right)_{\min }$, occurs at $X^{*}=0.43$, but not for the Vane MVGs $\left(X^{*}=1.29\right)$. For the baseline case with $\eta=15^{\circ}$, there is a distinctive kink at $X^{*} \approx 2$, which indicates SIBLS [23]. The pressure plateau moves downstream if MVGs are used. The location for $\left(P_{w} / P_{o}\right)_{\min }$ moves downstream because of trailing-edge expansion induced by MVGs [27]. $P_{w} / P_{o}$ has a lower amplitude downstream of the corner.

The $P_{w} / P_{o}$ distributions for $M=0.89$ are shown in Figure 9. The general flow characteristics are the same as those for $M=0.83$. For the baseline case, the low pressure region is longer $\left(X^{*}=0-3\right)$ or the onset of downstream compression occurs later than that for $M=0.83$. Bouhadji and Braza [28] studied transonic flow around an aerofoil in terms of the compressibility and viscous effects. For $M \approx 0.9$, they found that convection effect results in a sweep for perturbation action much farther downstream because of the highly hyperbolic character of the flow. When MVGs are installed, the low pressure region is observed at $X^{*}=0-6.4$. Therefore, all three types of MVGs produce stronger convection and force the shock farther downstream.

For the baseline case, the values for $\left(P_{w} / P_{o}\right)_{\min }$ or the local peak Mach number, $M_{\text {peak }}$, are reasonably well correlated with $\beta$ [4]. An increase in $\beta$ results in stronger expansion near the corner's apex. For $\beta=16.1\left(M=0.83\right.$ and $\left.\eta=13^{\circ}\right)$, Figure 10 shows that the value for $\left(P_{w} / P_{o}\right)_{\min }$ (or $\left.M_{\text {peak }}\right)$ is not significantly affected by the presence of MVGs. If $\beta$ increases, the presence of MVGs results in a decrease in $\left(P_{w} / P_{o}\right)_{\min }$ or an increase in $M_{\text {peak }}$. This is not the case for $\beta=22.6\left(M=0.83\right.$ and $\left.\eta=15^{\circ}\right)$ using Ramp MVGs. There is stronger expansion for $M=0.89$ for $\eta=13^{\circ}$ and $15^{\circ}$ (or $\beta=18.5$ and 26.1) because of convection effects. 


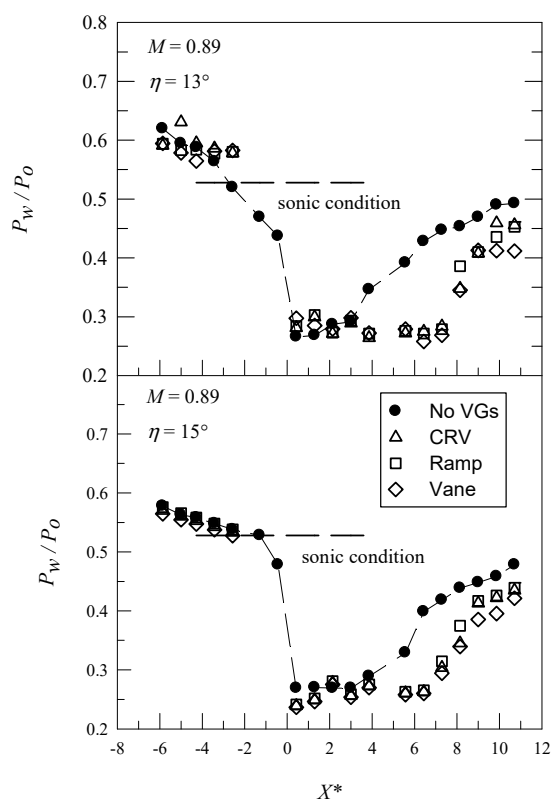

Figure 9. Mean surface pressure distributions for $M=0.89$.

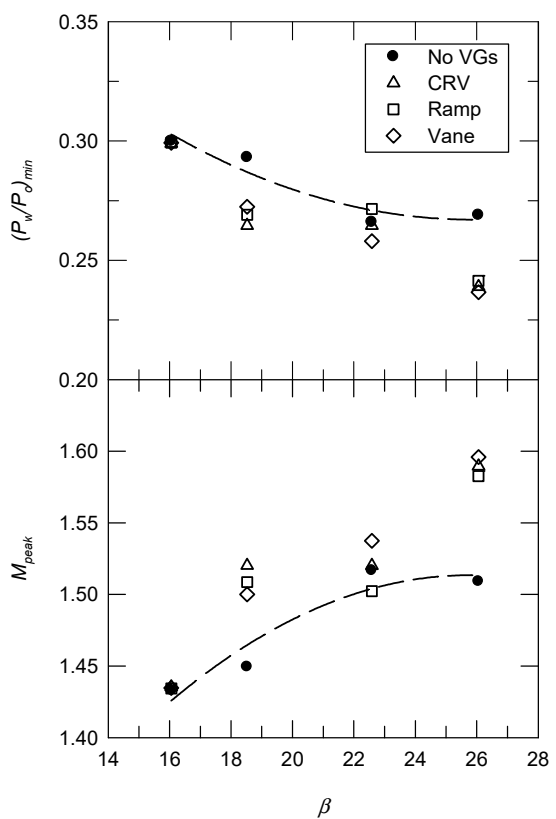

Figure 10. The effect of the presence of MVGs on flow expansion near the corner's apex.

\subsection{Surface Pressure Fluctuations}

The distribution of surface pressure fluctuations on a lifting surface corresponds to fluctuating loads, particularly during SIBLS and shock oscillation. For the baseline case for $M=0.83$, Figure 11 shows that the peak value for $\sigma_{p} / P_{w},\left(\sigma_{p} / P_{w}\right)_{\max }$, is observed at $X^{*}=2.14$, which corresponds to SIBLS [23]. The presence of MVGs results in a decrease in the value of $\left(\sigma_{p} / P_{w}\right)$ upstream and near the corner's apex $\left(X^{*}=-5.86-3.00\right)$ for $\eta=13^{\circ}$. 


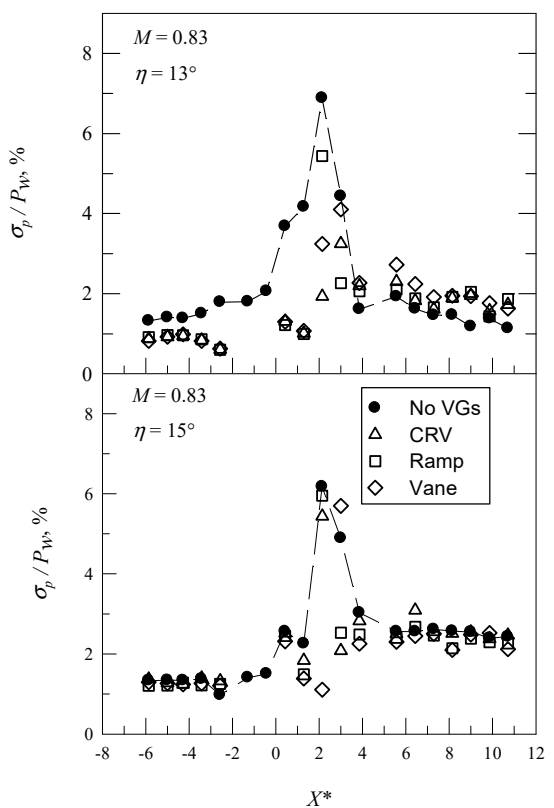

Figure 11. Surface pressure fluctuations for $M=0.83$.

Verma and Manisankar [18] showed that there is a reduction in $\left(\sigma_{p} / P_{w}\right)_{\max }$, when MVGs are present. This is also true for the test configuration in this study. If Vane MVGs are used, the location of $\left(\sigma_{p} / P_{w}\right)_{\max }$ moves downstream, which corresponds to $\left(P_{w} / P_{o}\right)_{\text {min }}$ (or $M_{\text {peak }}$ ) in Figure 8. For $\eta=15^{\circ}$, the effect of MVGs is less significant. For $M=0.89$, the distributions of surface pressure fluctuations are shown in Figure 12. These are similar to those for $M=0.83$, but the location of $\left(\sigma_{p} / P_{w}\right)_{\max }$ moves farther downstream $\left(X^{*}=3.86\right)$, which corresponds to the $P_{w} / P_{o}$ distributions that are shown in Figure 9.

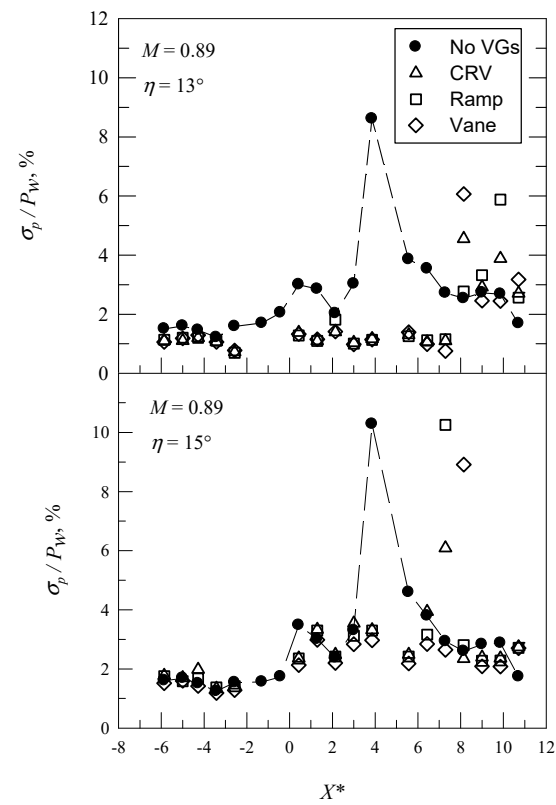

Figure 12. Surface pressure fluctuations for $M=0.89$.

The variation in $\left(\sigma_{p} / P_{w}\right)_{\max }$ with/without MVGs is shown in Figure 13. For the baseline case, $\left(\sigma_{p} / P_{w}\right)_{\max }$ is mainly associated with SIBLS and shock oscillation. The value of $\left(\sigma_{p} / P_{w}\right)_{\max }$ decreases if MVGs are used. For CRV MVGs, the primary flow passes through the split of the counter vanes and interacts with the flow that develops from each half of the device. The counter-rotating vortices are larger and stronger or flow becomes 
highly three-dimensional. There is a similar mechanism for Ramp MVGs. For the baseline case, extensive SIBLS occurs for $\beta \approx 20$ [4]. In Figure 13, the data for $\beta=18.5$ do not follow the global trend because of the presence of MVGs on $M_{\text {peak }}$ and shock oscillation. This determines that the effectiveness of MVGs depends on the size of separation bubble.

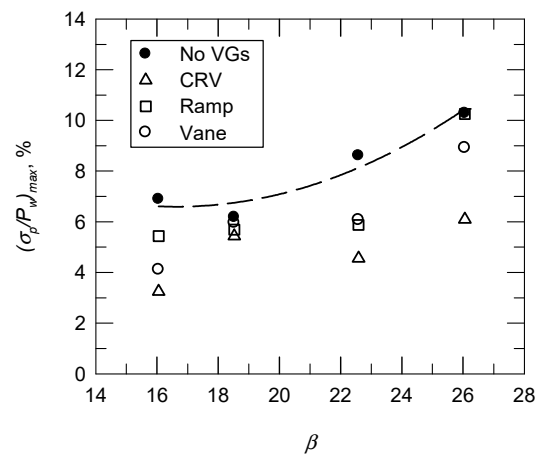

Figure 13. The effect of MVGs on peak pressure fluctuations.

Delery [29] showed that separation appears and develops more slowly and more progressively in three-dimensional flows than in two-dimensional flows. In terms of $\left(\sigma_{p} / P_{w}\right)_{\max }$ (Figure 13), CRV MVGs are the most effective at control for SIBLS. Ramp MVGs are less effective because the vortices are weaker and there is rapid lift-off from the surface [27]. Lee and Loth [30] determined that streamwise vortices are sustained at greater Mach numbers. The onset of flow separation occurs farther downstream for $M=0.89$ but the MVGs are still effective to mitigate surface pressure fluctuations.

\subsection{Surface Oil Flow Visualization}

Oil-flow visualization technique was used to determine the surface flow pattern. Examples for $\eta=13^{\circ}$ and $M=0.83$ with/without MVGs are shown in Figure 11. For the baseline case (Figure 14a), the surface flow pattern is primarily two-dimensional, in that there is little variation in the separation and reattachment locations in the spanwise direction. Separation and reattachment respectively occur at $X^{*} \approx 2.14$ and 8.14 (or the normalized separation length, $L^{*}=L / \delta$, of 6.00 ).

The presence of MVGs results in a corrugated pattern for separation and reattachment, which is most significant for CRV and Ramp types because there are counter-rotating vortices. There are sharper spikes than for Vane MVGs. This agrees with the results of the study by Lee et al. [31]. Figure 14 is used to determine the mean position for separation and reattachment.

The presence of MVGs delays flow separation, particularly for the CRV type. The respective mean separation and reattachment locations are at $X^{*}=4.71$ and 9.86 (or $\left.L^{*}=5.15\right)$, as shown in Figure 14b. For all MVGs, mean separation occurs slightly farther downstream. Mean reattachment for Ramp MVGs occurs farther upstream. A plot of $L^{*}$ versus $M_{\text {peak }}$ is shown in Figure 15. Ramp MVGs result in a greater reduction in $L^{*}$ and Vane MVGs has the least effect. 

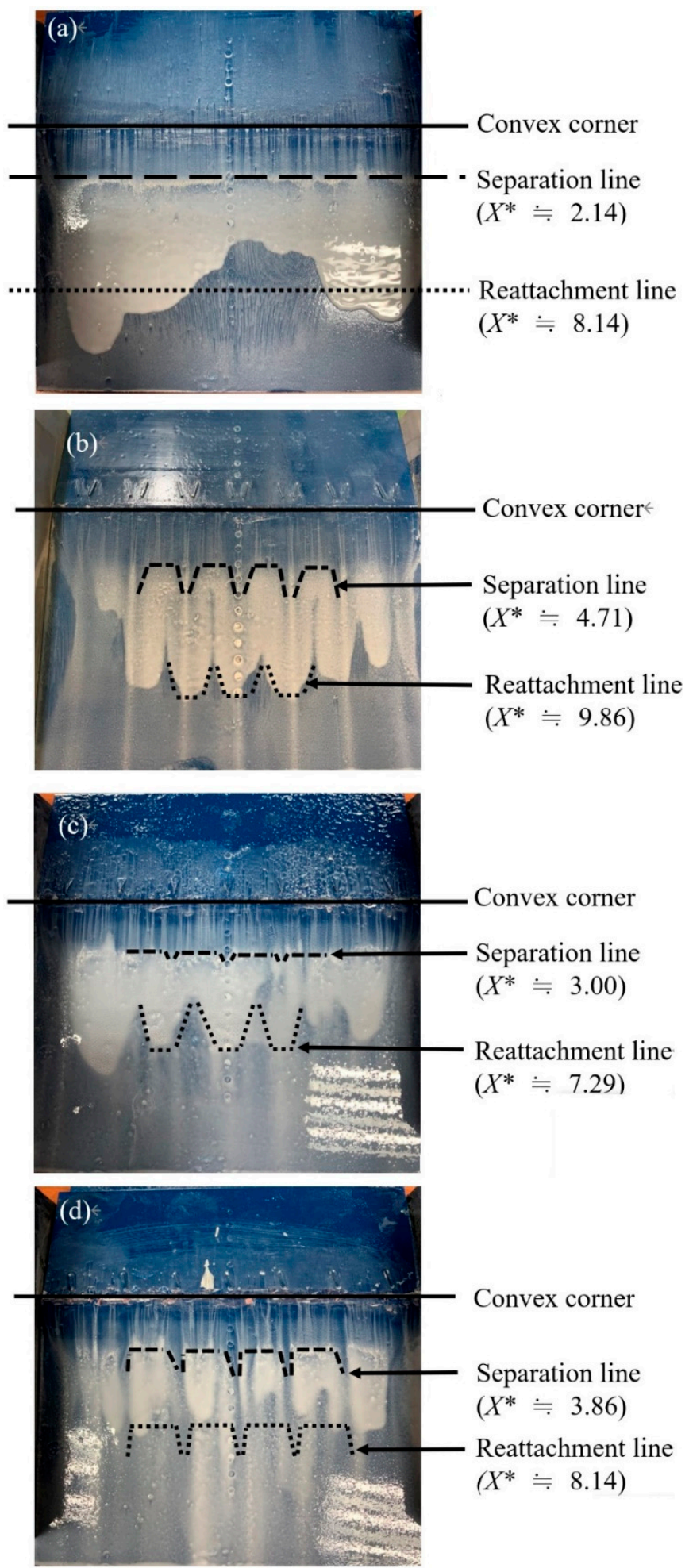

Figure 14. Surface oil flow visualization for $\eta=13^{\circ}$ and $M=0.83$ : (a) no MVGs, (b) CRV type, (c) Ramp type and (d) Vane type. 


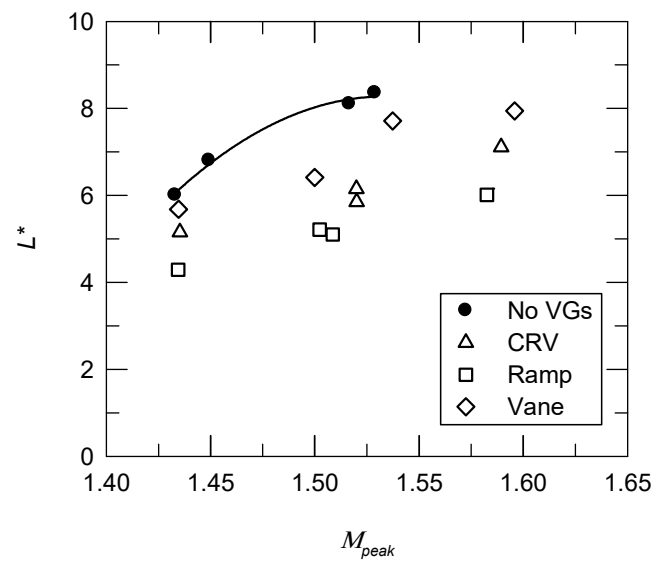

Figure 15. Normalized separation length versus peak Mach number.

\subsection{Self-Sustained Shock Oscillation}

THM was used to determine $f_{s}$ with/without MVGs, as shown in Figure 16. For the baseline case, the value of $f_{s}(640-1320 \mathrm{~Hz})$ decreases as $M_{\text {peak }}$ increases [4]. There is a significant reduction in the value of $f_{s}$ when MVGs are installed, particularly for the CRV type. This is similar to the variation in $\left(\sigma_{p} / P_{w}\right)_{\max }$. The data also shows that the decrease depends on $M$ rather than $\eta$. The respective values for the CRV type for $f_{s}$ are $535 \mathrm{~Hz}$ and $70 \mathrm{~Hz}$ for $M=0.83$ and 0.89 .

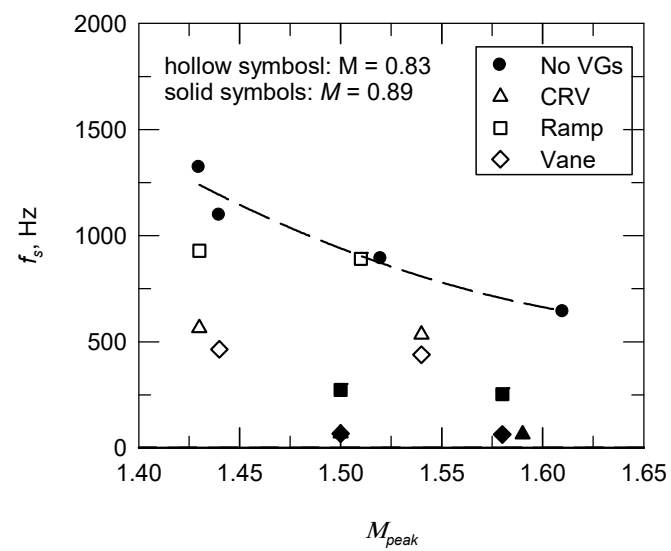

Figure 16. Shock zero-crossing frequency with/without MVGs.

Gonsalez and Dolling [32] showed that the peak $f_{s}$ is nondimensionalized in the form of the Strouhal number with the intermittent region length as the characteristic length scale, whose value lies within the range of $0.01-0.03$ for compression ramp and blunt-fin interactions. $L$ is used as the characteristic length scale in this study. The Strouhal number $S t\left(=f_{s} L / U_{p}\right)$ is determined, as shown in Figure 17, where $U_{p}$ is the peak velocity. The values range from 0.12 to 0.09 for the baseline case, when there is an increase in $M_{\text {peak }}$. The presence of MVGs stabilizes the shock, so the value of $S t$ is smaller, less than 0.06 . Therefore, shock oscillation is damped, particularly for $M=0.89(S t<0.02)$. 


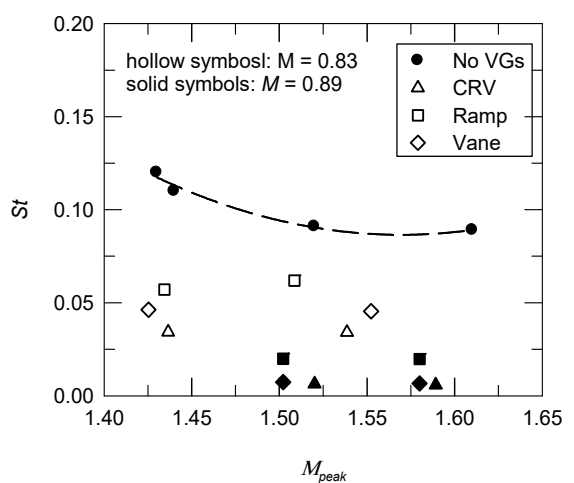

Figure 17. St with/without MVGs.

\section{Conclusions}

This study conducts experiments involving transonic convex-corner flow $(M=0.83$ and $0.89)$ with/without the presence of MVGs $\left(h^{*}=0.2\right)$. The mean/fluctuating surface pressure distributions, the local peak Mach number, the peak pressure fluctuations and the shock oscillation $\left(f_{s}\right.$ and $\left.S t\right)$ are determined. The presence of MVGs induces streamwise vortices, so there is a greater expansion wave near the corner's apex (the shock and compression move downstream) and flow separation is delayed. This is also true for the peak pressure fluctuations and the $f_{s}$. Shock oscillation is reduced, particularly for $M=0.89$.

The surface oil flow pattern for CRV MVGs is three-dimensional and the counterrotating vortices delay flow separation. For all MVGs, mean separation occurs farther downstream. Mean reattachment for Ramp MVGs occurs farther upstream. The Ramp MVGs produce the shortest separation length. The presence of MVGs is beneficial for deflected trailing-edge flaps as a high-lift device at transonic speeds.

Author Contributions: Conceptualization, K.-M.C. and K.-C.C.; methodology, K.-C.S.; formal analysis, K.-C.S.; resources, K.-M.C.; data curation, K.-C.S.; writing-original draft preparation, K.-C.S.; writing-review and editing, K.-M.C. and K.-C.C.; funding acquisition, K.-M.C. All authors have read and agreed to the published version of the manuscript.

Funding: This research was funded by the Ministry of Science and Technology, Taiwan, grant number MOST 107-2212-E-006-115-MY3.

Acknowledgments: The authors are grateful for the technical support of the ASTRC/NCKU technical staff.

Conflicts of Interest: The authors declare no conflict of interest.

\section{Nomenclature}

$f_{s} \quad$ shock zero-crossing frequency

$h \quad$ height of vortex generator

$h^{*} \quad$ normalized height of vortex generator, $h / \delta$

$L \quad$ mean separation length

$L^{*} \quad$ normalized mean separation length

$M \quad$ freestream Mach number

$M_{\text {peak }}$ peak Mach number

$P_{0} \quad$ stagnation pressure 


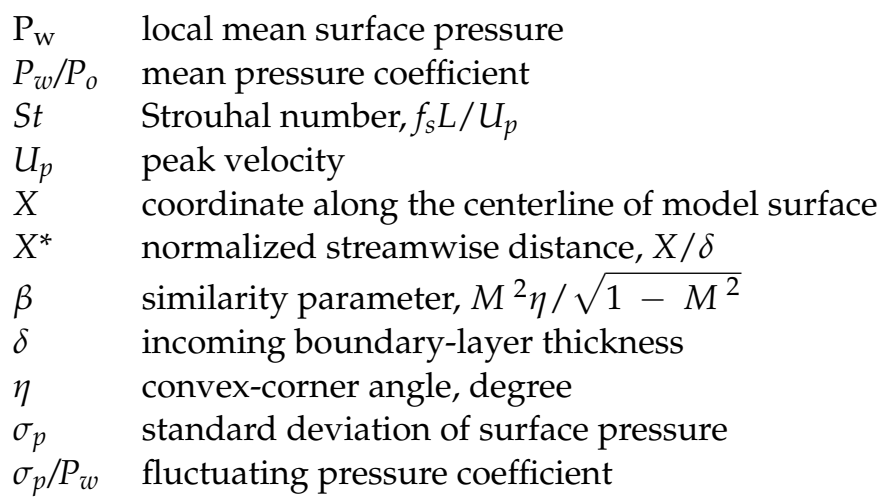

\section{References}

1. Szodruch, J.; Hilbig, R. Variable wing camber for transport aircraft. Prog. Aerosp. Sci. 1998, 25, 297-328. [CrossRef]

2. Liu, X.; Squire, L.C. An investigation of shock/boundary layer interactions on curved surfaces at transonic speeds. J. Fluid Mech. 1998, 187, 467-486. [CrossRef]

3. Chung, K.M. Unsteadiness of transonic convex-corner flows. Exp. Fluids 2004, 37, 917-922. [CrossRef]

4. Chung, K.M.; Chang, P.H.; Chang, K.C. Flow similarity in compressible convex-corner flows. AIAA J. 2012, 50, 985-988. [CrossRef]

5. Chung, K.M.; Lee, K.H.; Chang, P.H. Low-frequency shock motion in transonic convex-corner flows. AIAA J. 2017, 55, 2109-2112.

6. Ferrero, A. Control of a supersonic inlet in off-design conditions with plasma actuators and bleed. Aerospace 2020, 7, 32. [CrossRef]

7. Sun, Z.Z.; Miao, X.; Jaqadeesh, C. Experimental investigation of the transonic shock-wave/boundary-layer interaction over a shock-generation bump. Phys. Fluids 2020, 32, 106102. [CrossRef]

8. Huang, W.; Wu, H.; Yang, Y.G.; Yan, L.; Li, S.B. Recent advances in the shock wave/boundary layer interaction and its control in internal and external flows. Acta Astronaut. 2020, 174, 103-122. [CrossRef]

9. Gaqeik, M.; Nies, J.; Klioutchnikov, I.; Oliver, H. Pressure wave damping in transonic airfoil flow by means of micro vortex generators. Aerosp. Sci. Technol. 2018, 81, 65-77. [CrossRef]

10. Gao, W.Z.; Chen, J.; Liu, C.H.; Li, Z.F.; Yang, J.M.; Zeng, Y.S. Effects of vortex generators on unsteady unstarted flows of an axisymmetric inlet with nose bluntness. Aerosp. Sci. Technol. 2020, 104, 106021. [CrossRef]

11. Chung, K.C.; Su, K.C.; Chang, K.C. The effect of vortex generators on shock-induced boundary layer separation in a transonic convex-corner flow. Aerospace 2021, 8, 157. [CrossRef]

12. Baqheri, H.; Mirjalily, S.A.A.; Oloomi, S.A.A.; Salimpour, M.R. Effects of micro-vortex generators on shock wave structure in a low aspect ratio duct. numerical investigation. Acta Astronaut. 2020, 178, 616-624. [CrossRef]

13. Baydar, E.; Lu, F.K.; Slater, J.W. Vortex generators in a two-dimensional external-compression supersonic inlet. J. Prop. Power 2018, 34, 521-538. [CrossRef]

14. Lin, J.C. Review of research on low-profile vortex generators to control boundary-layer separation. Prog. Aerosp. Sci. 2002, 38, 389-420. [CrossRef]

15. Lin, J.C.; Robinson, S.K.; Mcghee, R.J.; Valarezo, W.O. Separation control on high-lift airfoils via micro-vortex generators. J. Aircr. 1994, 31, 1317-1323. [CrossRef]

16. Panaras, A.G.; Lu, F.K. Micro-vortex generators for shock wave/boundary layer interactions. Prog. Aerosp. Sci. 2015, 74, 16-47. [CrossRef]

17. Titchener, N.; Babinsky, H. A review of the use of vortex generators for mitigating shock-induced separation. Shock Waves 2015, 25, 473-494. [CrossRef]

18. Verma, S.B.; Manisankar, C. Assessment of various low-profile mechanical vortex generators in controlling a shock-induced separation. AIAA J. 2017, 55, 2228-2240. [CrossRef]

19. Jenkin, L.; Gorton, S.A.; Anders, S. Flow Control Device Evaluation for an Internal Flow with an Adverse Pressure Gradient; AIAA Paper 2002-0266; AIAA: Reno, NV, USA, 2002.

20. Bur, R.; Coponet, D.; Carpels, Y. Separation control by vortex generator devices in a transonic channel flow. Shock Waves 2009, 19, 521-530. [CrossRef]

21. Babinsky, H.; Li, Y.; Pitt Ford, C.W. Micro-ramp control of supersonic oblique shock-wave/boundary-layer interactions. AIAA J 2009, 47, 668-675. [CrossRef]

22. Lu, F.K. Visualization of supersonic flow around a sharp-edged, sub-boundary-layer protuberance. J. Visual. 2015, 18, 619-629. [CrossRef]

23. Chung, K.M. Investigation on transonic convex-corner flows. J. Aircr. 2002, 39, 1014-1018. [CrossRef]

24. Chung, K.M.; Miau, J.J. Calibration of ASTRC/NCKU $600 \mathrm{~mm} \times 600 \mathrm{~mm}$ transonic wind tunnel. In Proceedings of the 33rd AIAA Aerospace Science Meeting and Exhibit, Reno, NV, USA, 9-12 January 1995.

25. Bies, D.A. Flight and Wind Tunnel Measurements of Boundary Layer Pressure Fluctuations and Induced Structural Response; NASA-CR626; Langley Research Center, National Aeronautics and Space Administration: Washington, DC, USA, 1966. 
26. Dolling, D.S.; Brusniak, L. Separation shock motion in fin, cylinder, and compression ramp induced turbulent interactions. AIAA J. 1989, 27, 734-742. [CrossRef]

27. Holden, H.A.; Babinsky, H. Vortex generators near shock/boundary layer interactions. In Proceedings of the 42nd AIAA Aerospace Sciences Meeting and Exhibit, Reno, NV, USA, 10-13 January 2004.

28. Bouhadji, A.; Braza, M. Organised modes and shock-vortex interaction in unsteady viscous transonic flows around an aerofoil Part I: Mach number effect. Comput. Fluids 2003, 32, 1233-1260. [CrossRef]

29. Delery, J. Shock wave/turbulent boundary layer interaction and its control. Prog. Aerosp. Sci. 1985, 22, 209-280. [CrossRef]

30. Lee, S.; Loth, E. Effect of Mach number on flow past microramps. AIAA J. 2011, 49, 97-110. [CrossRef]

31. Lee, S.; Loth, E.; Babinsky, H. Normal shock boundary layer control with various vortex generator geometries. Comput. Fluids 2011, 49, 233-246. [CrossRef]

32. Gonsalez, J.C.; Dolling, D.S. Correlation of interaction sweepback effects on the dynamics of shock-induced turbulent separation. In Proceedings of the 31st AIAA Aerosp Sci Meeting and Exhibit, Reno, NV, USA, 11-14 January 1993. 